\section{P-68 STRIVING TO BECOME RESEARCH ACTIVE HOSPICES: THE STORY IN ONE REGION THROUGH ROLES AND COLLABORATION}

1,2,3,4 Joanne Leung, ${ }^{5}$ Charlotte Brigden. ${ }^{1}$ ellenor, Gravesend, UK; ${ }^{2}$ Heart of Kent Hospice, Aylesford, UK; ${ }^{3}$ Hospice in the Weald, Tunbridge Wells, UK; ${ }^{4}$ Wisdom Hospice, Rochester, UK; ${ }^{5}$ Pilgrims Hospices, Canterbury, UK

\subsection{6/bmjspcare-2016-001245.92}

Background The importance of undertaking clinical research has been reaffirmed by $95 \%$ of the public in a national survey (NIHR CRN, 2014). Yet, independent hospices often face challenges to conduct research partly due to different governance structures and procedures outside NHS organisations (Perkins et al., 2014). The Commission into the Future of Hospice Care highlighted the importance of research in hospices recommending how research could be implemented, including introduction of staff with 'research' in job titles and partnerships with universities (Payne et al., 2013).

Aims Five hospices within one region in England have begun implementing research through appointing a research facilitator and a research practitioner. They aim to

- facilitate collaboration between organisations

- enhance research awareness among staff

- support and develop new research projects within hospices

- engage in others' research.

Method The research posts have reached beyond their hospices. One belongs to the regional NIHR CRN and another has a formal link with a local university. In 2015, a local palliative care research group was set up, invited clinicians and academics to develop research and other related activity collaboratively. Both research facilitator and practitioner are members and facilitators of the group.

Results Reviewing the research strategy of the hospices has been initial work to reflect and standardise the research practices across hospices. Abundant information on research training, funding opportunities and research studies has also been widely shared amongst hospices. Excitingly, an upcoming annual local research day, the research group and research posts have allowed hospices to work collectively.

Conclusion Work within and between hospices is a stimulating opportunity to include wider pool of patients in research, extending the audience to comprehend the importance of research and increasing the evidence base for developing palliative care services. It is exhilarating to see research continuing to flourish across the region.

\section{P-69 PRIORITY SETTING FOR END-OF-LIFE CARE}

${ }^{1}$ Peter Mellor, ${ }^{2}$ Gunn Grande, ${ }^{2}$ Janet Diffin, ${ }^{1}$ Michael Spence, ${ }^{1}$ Rebecca Spencer. ${ }^{1}$ NIHR CLAHRC Greater Manchester (based at Salford Royal NHS Foundation Trust); ${ }^{2}$ The University of Manchester

\subsection{6/bmjspcare-2016-001245.93}

Introduction End-of-life (EoL) care is an under-researched area. It is vital to focus on the priorities that are likely to bring the most benefit to patients, carers, and their families. This project set out to identify the EoL research priorities of healthcare professionals (HCPs) and carers in Greater Manchester.

Approach Following a scoping exercise of EOL services in Greater Manchester, comprising of semi-structured interviews with HCPs, CLAHRC GM identified six topics within the 10 national EoL research priorities outlined by PeolcPSP. The topics identified were those that we have the capability to deliver research in:

1. Education and knowledge for staff and carers

2. Access to 24 hour care and support

3. Equitable Access

4. Advance care planning

5. Care at home

6. Continuity of care

CLAHRC GM held a priority setting event to consult with 32 HCPs and also held separate consultations with 26 carers facilitated by local carer groups from Manchester, Salford, and Bury during which HCPs and carers were asked to select their important priorities from the six topics.

Outcomes Combining carer and HCP responses, the topics of shared priority were:

- Advance care planning (62\% carers, 56\% HCPs)

- Access to 24-hour care (65\% carers, 50\% HCPs)

- Education and knowledge for staff and carers $(73 \%$ carers, $34 \%$ HCPs)

Whilst all topics were generally viewed as important by carers, there was more emphasis placed on training and education as the main priority whilst HCPs focused more on advance care planning.

HCPs also identified important research questions within their chosen topics.

Next steps The shared priorities will be used to develop the research questions identified by HCPs and, through further carer input, will shape the EoL research delivered across Greater Manchester over the next few years.

\section{P-70 DEVELOPING A HOSPICE RESEARCH CULTURE: LESSONS FROM A RANDOMISED TRIAL WITH 11 ORGANISATIONS}

${ }^{1}$ Catherine Walshe, ${ }^{1}$ Steven Dodd, ${ }^{1}$ Guillermo Perez Algorta, ${ }^{2}$ Matthew Hill, ${ }^{2}$ Nick Ockenden, ${ }^{1}$ Sheila Payne, ${ }^{1}$ Nancy Preston. 'International Observatory on End of Life Care, Lancaster, UK: ${ }^{2}$ Institute for Volunteering Research, NCVO, London, UK

\subsection{6/bmjspcare-2016-001245.94}

Background The Hospice UK report 'Research in palliative care' has led to an increase in hospices wanting to become more research active. However, to do this they often need a research project to catalyse this, and ideally support from palliative care research institutions.

Aim To report on the research experiences of 11 palliative care units who were partners in a randomised trial of befriending services

Methods A wait-list controlled trial with nested qualitative case studies investigating volunteer delivered befriending services across 11 sites. Patients $(n=195)$ were estimated to be in their last year of life and randomly allocated to receive the befriending intervention immediately or after a four week wait. We report on the skills developed by non-clinical hospice staff who were responsible for site trial management and informed consent procedures.

Results Training for this role included innovative face-to-face role play workshops, access to good clinical practice education, and virtual site visits to check procedural compliance and address concerns. Trial initiation issues included acknowledging site staff design concerns and involving them in developing the trial 
protocol, documentation and research procedures. Areas that required acknowledgement and response during the trial included providing support to address issues of informed consent and eligibility, understanding the impact of the 'wait' allocation on participants, staff and volunteers, and integrating volunteer management and feedback into trial procedures. At subsequent feedback sessions, staff moved from being research naive to research active making recommendations about the design of future studies.

Conclusions For most this was the first time they had been involved in research yet they embraced this and developed research skills for future studies. The trial acted as a catalyst for training of staff who were able to contribute to running a rigorous and ethical wait-list trial. Funded by the UK Cabinet Office.

\section{P-71 MAKING CHILDREN'S HOSPICES RESEARCH-READY: A CRN WEST MIDLANDS' INITIATIVE}

Krys Castro-Foskett, Hilary Shepley, Linda Porter, Katharine Lewney, Sharon Kempson. CRN West Midlands Children's Specialty

\subsection{6/bmjspcare-2016-001245.95}

Making children's hospices research-ready: A CRN West Midlands' initiative builds on the Payne Commission report in 2013 'Research in palliative care: can hospices afford not to be involved?' and recognising the opportunity in the CRN: WM region to engender research in children's hospices, engagement with the following hospices: Donna Louise Hospice, Acorns Hospice in Selly Oak and Hope House in Oswestry has started, in order to scope the needs for bringing research to this setting. Introductory and planning meetings with national key players were held and we also took part in initiatives eg Tweetfest to raise the profile of research in hospices, as well as gain advice and suggestions from key people nationally via Twitter. Next steps include expansion of GCP training, and providing paediatric communication and consent and PI masterclass courses to staff in children's hospices, in order to give the staff the practical skills to run research studies in children's hospices. CRN WM workforce development team will be involved in creating a bespoke training package for individual children's hospices, tailored to their need to be research-ready. These initiatives firmly link the clinical research network with the hospice staff forging good working relationships and ensuring that the research skills are acquired in readiness for opening the first paediatric hospice study.

\section{P-72 BUILDING RESEARCH CAPACITY TO ADDRESS THE PUBLIC HEALTH NEED FOR PALLIATIVE CARE}

Tara Murphy, Suzanne Guerin. All Ireland Institute of Hospice and Palliative Care, Dublin, Ireland

\subsection{6/bmjspcare-2016-001245.96}

Background The growing demand to address significant public health issues such as ageing populations, changing patterns of chronic diseases, individuals living longer with life-limiting conditions and so on increases the pressure on researchers to produce high quality, clinically relevant, innovative palliative care research so as to improve the quality of life for service users and carers.

Aim All Ireland Institute of Hospice and Palliative Care (AIIHPC) believes that one way to meet this demand is through research capacity building which is being taken forward via the Institute's Palliative Care Research Network.

Method Capacity building is defined as a "process where individuals, groups, networks, organisation and the wider community are encouraged and facilitated in enhancing their knowledge and skills so as to increase their ability to perform innovative and high quality research" (Moley and Seale, 2009). It is a major component of large national and international funding that seek to enhance and develop research expertise and leadership in specific health areas, for example, Irish Health Research Board, Public Health Agency (Northern Ireland), Horizon 2020, Canadian Institutes of Health Research).

Conclusion AIIHPC's Palliative Care Research Network is taking forward a multi-tiered approach to research capacity building ranging from awareness-raising of research, to providing more extensive support to those who want to progress elements of palliative care research, to providing tailored support and leadership opportunities for more advanced researchers.

\section{P-73 INTERDISCIPLINARY RESEARCH IN PALLIATIVE CARE: WHEN ACTIONS SPEAK LOUDER THAN WORDS}

Tara Murphy, Suzanne Guerin. All Ireland Institute of Hospice and Palliative Care, Dublin, Ireland

\subsection{6/bmjspcare-2016-001245.97}

Multidisciplinary research is characterised by two or more disciplines coming together to address the same issue or area of interest from their respective paradigms. Interdisciplinary research arguably moves several steps further to bring two or more distinct academic fields together, integrating theory, methodology, terminology and/or data to address critical and complex health issues (National Institutes of Health, 2007). A range of environmental, group and individual factors have been found to influence interdisciplinary working including organisational commitment, availability of resources, effective communication, strong leadership, mutual trust between partners, flexibility in addition to specific scholarly competencies (Aboelala et al., 2007; Gebbie et al., 2008). Porter et al., (2012) describe the correlation between funded interdisciplinary networking and scholarly impact noting that research articles arising from interdisciplinary research are more likely to be published in high impact journals and be highly cited.

This paper aims to provide a high-level overview of the highlights and challenges to adopting an interdisciplinary research approach in palliative care drawing on Irish, European and international experiences.

\section{Patient Care}

\section{P-74 HOW IMPLEMENTING THE FOUNDATION MODULES FOR PRODUCTIVE WARD HAS IMPROVED EFFICIENCY, SAFETY AND MORALE WITHIN THE INPATIENT UNIT}

Wendy Pepper, Dawn Griffiths, Kate Anderson, Helen Nutting, Alison Harris, Karen Tudge, Lynne Gilfillan, Lynne Perkins. Dorothy House Hospice Care, Bradford-On-Avon, UK

\subsection{6/bmjspcare-2016-001245.98}

Introduction The inpatient unit has introduced the three foundation modules from the Productive Ward programme, Patient Status at a Glance, The Well Organised Ward and Knowing How we 